\title{
Generalized Ratio and Product Methods of Estimation in Survey Sampling
}

\author{
Housila P. Singh \\ School of Studies in Statistics \\ Vikram University, Ujjain, India \\ hpsujn@gmail.com \\ Ramkrishna S. Solanki \\ School of Studies in Statistics \\ Vikram University, Ujjain, India \\ ramkssolanki@gmail.com
}

\begin{abstract}
This paper introduces some generalized ratio and product methods of estimation for estimating the population total Y. In addition to many Srivenkataramana and Tracy (1979) estimators are shown as members of the proposed estimators. The properties of the suggested estimators have been studied and their merits are examined through numerical illustration.
\end{abstract}

Keywords: Study variate, Auxiliary variate, Bias, Mean squared error.

\section{Introduction}

The use of auxiliary information has been dealt at great length for improving estimators in sample surveys. Ratio and product methods of estimation are good examples in this context. When there is a positively or negatively high correlation between the study variate and the auxiliary variate, it is hard to improve on these methods. In recent years considerable attempts have been made by various authors to increase precision of the conventional ratio and product methods of estimation by using suitable transformation on auxiliary variable $x$ with the help of certain known parameters of the auxiliary variable say $C_{x}$ (coefficient of variation), $\sigma_{x}$ (standard deviation), $\beta_{2}(x)$ (coefficient of kurtosis), $\rho$ (correlation coefficient between the study variable $y$ and the auxiliary variable $x$ ) and $\Delta=\left\{\beta_{2}(x)-\beta_{1}(x)-1\right\}$, where $\beta_{1}(x) \quad$ (coefficient of skewness) see Srivenkataramana and Tracy (1980), Bandyopadhyay (1980), Sisodia and Dwivedi (1981), Upadhyaya and Singh (1999), Singh (2003), Singh and Tailor (2003), Singh et al. (2004), Kadilar and Cingi (2006), Khoshnevisan et al. (2007), Gupta and Shabbir (2008), Singh et al. (2008), Singh and Agnihotri (2008) and Koyuncu and Kadilar (2010). Mohanty and Sahoo (1995) considered the transformation on the auxiliary variate $\mathrm{x}$ using available knowledge on the $\mathrm{x}_{\mathrm{m}}$ (the minimum value) and $x_{M}$ (the maximum value) of the auxiliary variate $x$. We also note that some interesting work based on multiphase sampling have been carried by Hanif et al. (2010) and Butt et al. (2010). 
It is further noted that there are some transformations available based on the unknown constant which requires a good guess of the value of $k=\rho\left(C_{y} / C_{x}\right)$, where $C_{y}$ and $C_{x}$ are the coefficients of variation of $y$ and $x$ respectively in the population, see Reddy (1974), Srivenkataramana and Tracy (1980).

It may happen that the correlation ' $\rho$ ' between study variate $y$ and auxiliary variate $\mathrm{x}$ is just moderate. There is a need for estimators or combination of estimators for dealing with such cases. Srivenkataramana and Tracy (1979) have made an attempt in this direction, built around the idea that estimating the population total is essentially equivalent to estimating the total corresponding to the nonsampling unit, since the corresponding to the sample units is known once the sample is drawn and measurements are made on it.

The objective of this paper is to suggest a generalized ratio and product methods of estimation using a general transformation on the auxiliary variable $\mathrm{x}$. We have identified several estimators for population total $Y$ of the study variate $y$ and shown that the estimators reported by Srivenkataramana and Tracy (1979) are members of the suggested estimators. The properties of the suggested estimators are studied and their merits are examined through empirical study.

\section{Estimator for the case of positive correlation}

Consider a finite population with $\mathrm{N}$ units $\left(\mathrm{U}_{1}, \mathrm{U}_{2}, \ldots, \mathrm{U}_{\mathrm{N}}\right)$. The variate of interest (study) $y$ and the auxiliary variate $x$ related to $y$ assume real non-negative values $\left(Y_{i}, X_{i}\right)$ on the unit $U_{i}, i=1,2, \ldots, N$. This non-negativity condition is met by almost all sample survey universes. The population total $X=\sum_{i=1}^{N} X_{i}$ is known and is positive and the total $Y=\sum_{i=1}^{N} Y_{i}$ is to be estimated. Such situations are quite frequent in sample surveys. A simple random sample of $n \leq N$ units is drawn without replacement from the population under study and observations are made on the units selected. The totals $y, x$-values for the $n$ units in the sample are $Y^{(1)}, X^{(1)}$ and the similar total for the $(N-n)$ units not included in the sample are $Y^{(2)}$ and $X^{(2)}$. Then obviously

$$
Y=Y^{(1)}+Y^{(2)}
$$

and

$$
X=X^{(1)}+X^{(2)}
$$

for any possible sample.

In view of (2.1), essentially an estimate of $Y^{(2)}$ is sufficient for estimating $Y$ since $Y^{(1)}$ can be computed from the sample.

Let

$$
\hat{Y}=\frac{N}{n} Y^{(1)},
$$




$$
\hat{X}=\frac{N}{n} X^{(1)},
$$

be the (unbiased) sample expansion estimators of $Y$ and $X$ respectively, constructed from the sample. If $\rho$ is positively high then it is conventional to use the ratio method of estimation, where

$$
\hat{Y}_{R}=\hat{R} X=Y^{(1)}\left\{\frac{X}{X^{(1)}}\right\},
$$

is used as the estimator of population total $Y$. Here $\hat{R}=\hat{Y} / \hat{X}=Y^{(1)} / X^{(1)}$ estimates $\mathrm{R}=\mathrm{Y} / \mathrm{X}$.

Introducing the following transformation on the auxiliary variable $\mathrm{x}$ :

$$
Z_{i}=a X_{i}+b, i=1,2, \ldots, N \text {, }
$$

on the lines of Singh and Agnihotri (2008), we define the following ratio type estimator for population total $\mathrm{Y}$ as

$$
d_{r}=Y^{(1)} \frac{(a X+N b)}{\left(a X^{(1)}+n b\right)}
$$

where $(a, b)$ are positive constants which may be the known parameters of auxiliary variable $x$ such as $C_{x}, S_{x}, \beta_{1}(x), \beta_{2}(x), \rho$ and $\left.\Delta=\left\{\beta_{2}(x)-\beta_{1}(x)-1\right)\right\}$, see Singh and Agnihotri $(2008, p .43)$. Some members of the estimator $d_{r}$ are listed in Table 2.1.

Table 2.1: Some estimators of the population total $Y(=N \bar{Y})$ based on transformed auxiliary variable $x$.

\begin{tabular}{|l|c|c|}
\hline \multirow{2}{*}{ Estimator } & \multicolumn{2}{|c|}{ Values of constants } \\
\cline { 2 - 3 }$d_{1}=Y^{(1)}\left\{\frac{X+N C_{x}}{X^{(1)}+n C_{x}}\right\}$ & 1 & $C_{x}$ \\
{$[$ Sisodia and Dwivedi (1981)] } & $\rho$ & $C_{x}$ \\
\hline$d_{2}=Y^{(1)}\left\{\frac{X \rho+N C_{x}}{X^{(1)} \rho+n C_{x}}\right\}$ & $\rho$ & 1 \\
{$[$ Kadilar and Cingi (2006)] } & $\mathrm{Xp+N}$ \\
\hline$d_{3}=Y^{(1)}\left\{\frac{X}{X^{(1)} \rho+n}\right\}$ & $f$ & 1 \\
\hline$d_{4}=Y^{(1)}\left\{\frac{X f+N}{X^{(1)} f+n}\right\}$ & $f$ & $g$ \\
\hline$d_{5}=Y^{(1)}\left\{\frac{X f+N g}{X^{(1)} f+n g}\right\}$ & $g$ & 1 \\
\hline$d_{6}=Y^{(1)}\left\{\frac{X g+N}{X^{(1)} g+n}\right\}$ & & \\
\hline
\end{tabular}


Further we define the population total $Z=N \bar{Z}=(a X+N b)$ of the variable $z$. It can be defined as

$$
Z=Z^{(1)}+Z^{(2)}=\left\{a X^{(1)}+n b\right\}+\left\{a X^{(2)}+(N-n) b\right\}
$$

where $Z^{(1)}$ and $Z^{(2)}$ are the totals of $n$ units in the sample and $(N-n)$ units not included in the sample.

Let $\hat{Z}=\frac{N}{n} Z^{(1)}$ be the (unbiased) simple expansion estimator of the population total $Z$ of the variable $z$, computed from the sample.

Motivated by the identities (2.1) and (2.2), we consider the following estimators for population total $Y$ as

$$
\begin{aligned}
& d_{r(1)}=Y^{(1)}+\left\{\hat{Y}-Y^{(1)}\right\} \frac{Z^{(2)}}{g Z}=Y^{(1)}\left\{1+\frac{Z^{(2)}}{f Z}\right\}=Y^{(1)}\left[1+\frac{\left\{a X^{(2)}+(N-n) b\right\}}{f(a X+N b)}\right], \\
& d_{r(2)}=Y^{(1)}+\left\{\hat{Y}-Y^{(1)}\right\} \frac{Z}{Z}=Y^{(1)}\left\{1+\frac{g Z}{Z^{(1)}}\right\}=Y^{(1)}\left[1+\frac{g(a X+N b)}{\left\{a X^{(1)}+n b\right\}}\right],
\end{aligned}
$$

where $f=(n / N)$ is the sampling fraction and $g=(1-f)$.

For $(a, b)=(1,0), d_{r(1)}$ and $d_{r(2)}$ respectively reduce to

$$
\hat{Y}_{1}=Y^{(1)}\left\{1+\frac{X^{(2)}}{f X}\right\}
$$

and

$$
\hat{Y}_{2}=Y^{(1)}\left\{1+\frac{g X}{X^{(1)}}\right\}
$$

which are due to Srivenkataramana and Tracy (1979).

The expressions on the extreme right in (2.3) and (2.4) show the near-duality of the estimators $d_{r(1)}$ and $d_{r(2)}$. Here the unknown $Y^{(2)}$ has been estimated by $\left\{\hat{Y}-Y^{(1)}\right\}$ modified by the factor $\frac{Z^{(2)}}{g Z}$ or $\frac{Z}{\hat{Z}}$. Note that $Z^{(2)}$ is known once the sample is drawn and $Z^{(1)}$ is computed. Also

while

$$
\left.\operatorname{Cor}\left\{\hat{Y} Y-Y^{(1)}\right), X^{(2)}\right\}=\operatorname{Cor}\{(N-n) \bar{y},(X-n \bar{x})\}=-\rho
$$

$$
\operatorname{Cor}\left\{\left(\hat{Y}-Y^{(1)}\right), \hat{X}\right\}=\operatorname{Cor}\{(N-n) \bar{y}, N \bar{x}\}=\rho,
$$

where $\bar{y}$ and $\bar{x}$ are the sample mean for $y$ and $x$ respectively. The second component of $d_{r(1)}$ is of product-type since the two estimates there, viz. $\left\{\hat{Y}-Y^{(1)}\right\}$ and $Z^{(2)}$, are negatively correlated, where as the second component of $d_{r(2)}$ is of ratio-type, in view of the positive correlation between $\left\{\hat{Y}-Y^{(1)}\right\}$ and $\hat{X}$. 
A large number of estimators can be generated from the proposed estimators $d_{r(1)}$ and $d_{r(2)}$. The following set of estimators listed in Table 2.2 are members of the suggested estimators $d_{r(1)}$ and $d_{r(2)}$.

Table 2.2: Some members of the estimators $d_{r(1)}$ and $d_{r(2)}$

\begin{tabular}{|l|c|c|}
\hline \multirow{2}{*}{ Estimators } & \multicolumn{2}{|c|}{ Values of constants } \\
\cline { 2 - 3 }$d_{1(1)}=Y^{(1)}\left[1+\frac{\left\{X^{(2)}+(N-n) C_{x}\right\}}{f\left(X+N C_{x}\right)}\right]$ & 1 & $c_{x}$ \\
$d_{1(2)}=Y^{(1)}\left[1+\frac{g\left(X+N C_{x}\right)}{\left\{X^{(1)}+n C_{x}\right\}}\right]$ & 1 & $C_{x}$ \\
\hline$d_{2(1)}=Y^{(1)}\left[1+\frac{\left\{X^{(2)} \rho+(N-n) C_{x}\right\}}{f\left\{X^{(1)} p+N C_{x}\right\}}\right]$ & $\rho$ & $C_{x}$ \\
$d_{2(2)}=Y^{(1)}\left[1+\frac{g\left\{X \rho+N C_{x}\right\}}{\left\{X^{(1)} p+n C_{x}\right\}}\right.$ & $\rho$ & $C_{x}$ \\
\hline$d_{3(1)}=Y^{(1)}\left[1+\frac{\left\{X^{(2)} \rho+(N-n)\right\}}{f(X p+N)}\right]$ & $\rho$ & 1 \\
$d_{3(2)}=Y^{(1)}\left[1+\frac{g\{X \rho+N\}}{\left\{X^{(1)} p+n\right\}}\right]$ & $\rho$ & 1 \\
\hline$d_{4(1)}=Y^{(1)}\left[1+\frac{\left\{X^{(2)} f+(N-n)\right\}}{f\left\{X^{(1)} f+N\right\}}\right]$ & $f$ & 1 \\
$d_{4(2)}=Y^{(1)}\left[1+\frac{g\{X f+N\}}{\left\{X^{(1)} f+n\right\}}\right]$ & $f$ & 1 \\
\hline$d_{5(1)}=Y^{(1)}\left[1+\frac{\left\{X^{(2)} f+(N-n) g\right\}}{f(X f+N g)}\right]$ & $f$ & $g$ \\
$d_{5(2)}=Y^{(1)}\left[1+\frac{g(X f+N g)}{\left\{X^{(1)} f+n g\right\}}\right]$ & $f$ & $g$ \\
\hline$d_{6(1)}=Y^{(1)}\left[1+\frac{\left\{X^{(2)} g+(N-n)\right\}}{f\left\{X^{(1)} g+N\right\}}\right]$ & $g$ & 1 \\
$d_{6(2)}=Y^{(1)}\left[1+\frac{g\{X g+N\}}{\left\{X^{(1)} g+n\right\}}\right]$ & $g$ & 1 \\
\hline
\end{tabular}

In order to consider the efficiencies of these estimators in comparison to those of the unbiased estimator $\hat{Y}$, usual ratio estimator $\hat{Y}_{R}$, Srivenkataramana and 
Tracy (1979) estimators $\hat{Y}_{1}, \hat{Y}_{2}$, ratio-type estimators $d_{r}$ and $d_{r(j)}(j=1,2)$. The expressions for the bias and the mean square error (MSE) of the estimators are given in the following section.

\section{Biases and MSEs of the estimators $d_{r}$ and $d_{r(j)}(j=1,2)$}

To obtain the bias and MSE expressions of different estimators of the population total $Y$, we write

$$
\begin{aligned}
& \hat{Y}=Y\left(1+e_{0}\right), \\
& \hat{X}=X\left(1+e_{1}\right),
\end{aligned}
$$

such that

$$
E\left(e_{0}\right)=E\left(e_{1}\right)=0
$$

and

$$
V_{i j}=E\left(e_{0}^{i} e_{1}^{j}\right)=\frac{E\left\{(\hat{Y}-Y)^{i}(\hat{X}-X)^{j}\right\}}{Y^{i} Y^{j}} \text { (relative central moments), }
$$

where $(i, j)$ are non-negative integers.

Expressing $d_{r}, d_{r(1)}$ and $d_{r(2)}$ in terms of e's we have

$$
\begin{aligned}
& d_{r}=Y\left(1+e_{0}\right)\left(1+\lambda e_{1}\right)^{-1}, \\
& d_{r(1)}=Y\left(1+e_{0}\right)\left(1-\lambda f e_{1}\right), \\
& d_{r(2)}=Y\left(1+e_{0}\right)\left[f+g\left(1+\lambda e_{1}\right)^{-1}\right],
\end{aligned}
$$

where $\lambda=\frac{\mathrm{aX}}{(\mathrm{aX}+\mathrm{Nb})}$.

We assume that $\left|\lambda e_{1}\right|<1$ so that $\left(1+\lambda e_{1}\right)^{-1}$ is expandable in powers of $\lambda e_{1}$. Expanding the right hand sides of (3.1), (3.2) and (3.3) multiplying out and neglecting terms of e's having power greater than four, we have

$$
\begin{aligned}
& \left(d_{r}-Y\right) \approx Y\left[e_{0}-\lambda e_{1}+\lambda^{2} e_{1}^{2}-\lambda e_{0} e_{1}+\lambda^{2} e_{0} e_{1}^{2}-\lambda^{3} e_{1}^{3}+\lambda^{4} e_{1}^{4}-\lambda^{3} e_{0} e_{1}^{3}\right], \\
& \left(d_{r(1)}-Y\right)=Y\left[e_{0}-\lambda f e_{1}-\lambda f e_{0} e_{1}\right], \\
& \left(d_{r(2)}-Y\right) \approx Y\left[e_{0}-g\left(\lambda e_{1}+\lambda e_{0} e_{1}-\lambda^{2} e_{1}^{2}-\lambda^{2} e_{0} e_{1}^{2}+\lambda^{3} e_{1}^{3}+\lambda^{3} e_{0} e_{1}^{3}-\lambda^{4} e_{1}^{4}\right)\right]
\end{aligned}
$$

Taking expectation of both sides of (3.4), (3.5) and (3.6) we get the approximate expressions for the bias of the estimators $d_{r}, d_{r(1)}$ and $d_{r(2)}$ respectively as

$$
\begin{aligned}
& B\left(d_{r}\right)=Y\left[\lambda^{2} V_{02}-\lambda V_{11}+\lambda^{2} V_{12}-\lambda^{3} V_{03}+\lambda^{4} V_{04}-\lambda^{3} V_{13}\right] \\
& B\left(d_{r(1)}\right)=-f Y \lambda V_{11}, \\
& B\left(d_{r(2)}\right)=Y g\left[\lambda^{2} V_{02}-\lambda V_{11}+\lambda^{2} V_{12}-\lambda^{3} V_{03}+\lambda^{4} V_{04}-\lambda^{3} V_{13}\right]
\end{aligned}
$$


Squaring both sides of (3.5), (3.6), (3.7) and neglecting terms of e's having power greater four we have

$$
\begin{aligned}
\left(d_{r}-Y\right)^{2} \approx Y^{2}\left[e_{0}^{2}-2 \lambda e_{0} e_{1}+\lambda^{2} e_{1}^{2}-2 \lambda e_{0}^{2} e_{1}+4 \lambda^{2} e_{0} e_{1}^{2}-2 \lambda^{3} e_{1}^{3}\right. & \\
& \left.+3 \lambda^{3} e_{0}^{2} e_{1}^{2}-6 \lambda^{3} e_{0} e_{1}^{3}+3 \lambda^{4} e_{1}^{4}\right] \\
\left(d_{r(1)}-Y\right)^{2} \approx Y^{2}[ & \left.e_{0}^{2}+f^{2} \lambda^{2}\left(e_{1}^{2}+e_{0}^{2} e_{1}^{2}+2 e_{0} e_{1}^{2}\right)-2 f \lambda\left(e_{0} e_{1}+e_{0}^{2} e_{1}\right)\right] \\
\left(d_{r(2)}-Y\right)^{2} \approx Y^{2}[ & e_{0}^{2}+g\left\{g \lambda^{2} e_{1}^{2}-2 \lambda e_{0} e_{1}+2 \lambda^{2}(1+g) e_{0} e_{1}^{2}-2 \lambda e_{0}^{2} e_{1}-2 \lambda^{3} g e_{1}^{3}\right. \\
& \left.\left.+\lambda^{2}(g+2) e_{0}^{2} e_{1}^{2}-2 \lambda^{3}(1+2 g) e_{0} e_{1}^{3}+3 \lambda^{4} g e_{1}^{4}\right)\right]
\end{aligned}
$$

Taking expectation of both sides of (3.10), (3.11) and (3.12) we get the approximate expressions of the mean squared errors of the estimators $d_{r}, d_{r(1)}$ and $d_{r(2)}$ respectively as

$$
\begin{aligned}
& \operatorname{MSE}\left(d_{r}\right)=Y^{2}\left[V_{20}-2 \lambda V_{11}+\lambda^{2} V_{02}-2 \lambda V_{21}+4 \lambda^{2} V_{12}-2 \lambda^{3} V_{03}+3 \lambda^{3} V_{22}\right. \\
&\left.-6 \lambda^{3} V_{13}+3 \lambda^{4} V_{04}\right] \\
& \operatorname{MSE}\left\{d_{r(1)}\right\}=Y^{2}\left[V_{20}+f \lambda\left\{f \lambda V_{02}-2 V_{11}+2 \lambda f V_{12}-2 V_{21}+\lambda f V_{22}\right\}\right] \\
& \operatorname{MSE}\left\{d_{r(2)}\right\}=Y^{2}\left[V_{20}+g\left\{g \lambda^{2} V_{02}-2 \lambda V_{11}+2 \lambda^{2}(1+g) V_{12}-2 \lambda V_{21}-2 \lambda^{3} g V_{03}\right.\right. \\
&\left.\left.+\lambda^{2}(2+g) V_{22}-2 \lambda^{3}(1+2 g) V_{13}+3 \lambda^{4} g V_{04}\right\}\right]
\end{aligned}
$$

It may be remarked that the expressions (3.8) and (3.14) are exact. It follows from (3.9) that the estimator $d_{r(2)}$ always leads to a $100 f \%$ reduction in the absolute value of the bias relative to that of $d_{r}$.

For simple random or varying probability sampling with replacement or any scheme involving independent subsamples, $V_{i j}$ with $i+j>2$ are generally small, see Murthy [1967, pp.380-381]. Thus to have tangible idea about the biases and MSEs of the estimators $d_{r}, d_{r(1)}, d_{r(2)}$ and the estimators belonging to these estimators, we neglect the terms $\mathrm{V}_{\mathrm{ij}}$ with $\mathrm{i}+\mathrm{j}>2$ in (3.7)-(3.9) and (3.13)-(3.15).

$$
\begin{aligned}
& B\left(d_{r}\right) \approx Y\left(\lambda^{2} V_{02}-\lambda V_{11}\right), \\
& B\left\{d_{r(1)}\right\} \approx-Y f \lambda V_{11}, \\
& B\left\{d_{r(2)}\right\} \approx Y g\left(\lambda^{2} V_{02}-\lambda V_{11}\right)=g B\left(d_{r}\right), \\
& \operatorname{MSE}\left(d_{r}\right) \approx Y^{2}\left[V_{20}-2 \lambda V_{11}+\lambda^{2} V_{02}\right], \\
& \operatorname{MSE}\left\{d_{r(1)}\right\} \approx Y^{2}\left[V_{20}+f \lambda\left\{f \lambda V_{02}-2 V_{11}\right\}\right], \\
& \operatorname{MSE}\left(d_{r(2)}\right) \approx Y^{2}\left[V_{20}+g\left\{g \lambda^{2} V_{02}-2 \lambda V_{11}\right\}\right] .
\end{aligned}
$$


In case of simple random sampling without replacement (SRSWOR) $V_{11}=\left(\frac{g}{n}\right) k C_{x}^{2}, V_{20}=\left(\frac{g}{n}\right) C_{y}^{2}$ and $V_{02}=\left(\frac{g}{n}\right) C_{x}^{2}$ with $k=\rho\left(\frac{C_{y}}{C_{x}}\right)$ and the expressions (3.16)-(3.21) respectively reduce to

$$
\begin{aligned}
& B\left(d_{r}\right)=\left(\frac{g}{n}\right) \lambda Y(\lambda-k) C_{x}^{2}, \\
& B\left\{d_{r(1)}\right\}=-\left(\frac{g}{n}\right) f \lambda Y k C_{x}^{2}, \\
& B\left\{d_{r(2)}\right\}=\left(\frac{g^{2}}{n}\right) \lambda Y(\lambda-k) C_{x}^{2}=g B\left(d_{r}\right), \\
& \operatorname{MSE}\left(d_{r}\right)=\left(\frac{g}{n}\right) Y^{2}\left[C_{y}^{2}+\lambda C_{x}^{2}(\lambda-2 k)\right], \\
& \operatorname{MSE}\left\{d_{r(1)}\right\}=\left(\frac{g}{n}\right) Y^{2}\left[C_{y}^{2}+\lambda f C_{x}^{2}(\lambda f-2 k)\right], \\
& \operatorname{MSE}\left\{d_{r(2)}\right\}=\left(\frac{g}{n}\right) Y^{2}\left[C_{y}^{2}+\lambda g C_{x}^{2}(g \lambda-2 k)\right],
\end{aligned}
$$

\section{Efficiency comparison}

The reductions in the MSE of the estimator $d_{r(1)}$ or $d_{r(2)}$ is used in the place of $d_{r}$, up to second order moments, given by

$$
\begin{aligned}
& \operatorname{MSE}\left(d_{r}\right)-\operatorname{MSE}\left\{d_{r(1)}\right\}=\left(\frac{g^{2}}{n}\right) Y^{2} \lambda C_{x}^{2}[\lambda(1+f)-2 k], \\
& \operatorname{MSE}\left(d_{r}\right)-\operatorname{MSE}\left\{d_{r(2)}\right\}=f\left(\frac{g}{n}\right) Y^{2} \lambda C_{x}^{2}[\lambda(1+g)-2 k] .
\end{aligned}
$$

For $(k / \lambda)<(1 / 2)$ (where $\lambda$ is a known quantity) and usual sampling fractions the reduction given in (4.1) can be appreciable. The reduction given by (4.2) can be substantial for $\frac{k}{\lambda}$ even in the interval $\left(\frac{1}{2}, 1-\frac{f}{2}\right)$.

It is well known under SRSWOR that

$$
\operatorname{MSE}(\hat{Y})=\operatorname{var}(\hat{Y})=\left(\frac{g}{n}\right) Y^{2} C_{y}^{2} \text {. }
$$

From (3.23) and (4.3) we have

$$
\begin{aligned}
& \operatorname{MSE}\left\{d_{r(1)}\right\}-\operatorname{MSE}(\hat{Y})=\frac{g}{n} Y^{2} \lambda f C_{x}^{2}(\lambda f-2 k)>0, \quad \text { if } \\
& (k / \lambda)<(f / 2) .
\end{aligned}
$$


It follows from $(4.4)$ that for $(k / \lambda)<(f / 2)$ the use of the conventional estimator $\hat{Y}$ is to be preferred over the estimator $d_{r(1)}$.

Thus the guidelines for selecting among the estimators $\hat{Y}, d_{r}, d_{r(1)}$ and $d_{r(2)}$ using the minimum MSE criterion [see Srivenkataramana and Tracy (1979, p. 41)] are given in Table 4.1.

Table 4.1: The case of positive correlation

\begin{tabular}{|l|l|l|l|}
\hline \multicolumn{2}{|c|}{$f \leq \frac{1}{2}$} & \multicolumn{2}{c|}{$f>\frac{1}{2}$ or $g=(1-f)<\frac{1}{2}$} \\
\hline Interval for $\frac{k}{\lambda}$ & $\begin{array}{l}\text { preferred } \\
\text { estimator }\end{array}$ & \multicolumn{1}{|c|}{ Interval for $\frac{k}{\lambda}$} & $\begin{array}{l}\text { preferred } \\
\text { estimator }\end{array}$ \\
\hline$(1)$ & $(2)$ & $(3)$ & $(4)$ \\
\hline $0 \leq \frac{k}{\lambda} \leq \frac{f}{2}$ & $\hat{Y}$ & $0<\frac{k}{\lambda} \leq \frac{g}{2}$ & $\hat{Y}$ \\
\hline$\frac{f}{2}<\frac{k}{\lambda} \leq \frac{1}{2}$ & $d_{r(1)}$ & $\frac{g}{2}<\frac{k}{\lambda} \leq \frac{1}{2}$ & $d_{r(2)}$ \\
\hline$\frac{1}{2}<\frac{k}{\lambda} \leq 1-\frac{f}{2}$ & $d_{r(2)}$ & $\frac{1}{2}<\frac{k}{\lambda} \leq 1-\frac{g}{2}$ & $d_{r(1)}$ \\
\hline$\frac{k}{\lambda}>1-\frac{f}{2}$ & $d_{r}$ & $\frac{k}{\lambda}>1-\frac{g}{2}$ & $d_{r}$ \\
\hline
\end{tabular}

As the argument given in Srivenkataramana and Tracy (1979, p. 41) we mention that if a good guess of the interval which contain $\frac{k}{\lambda}$ ( $\lambda$ is a known quantity) can be made based on theory, accumulated experience and or/a scatter diagram for at least a part of current data then it is observed that the selection of the preferred estimator is not hard to make.

Putting $(a, b)=(1,0) \Rightarrow \lambda=1$ in (3.22) we get the MSE of the ratio estimator

$$
\hat{Y}_{R}=Y^{(1)} \frac{X}{X^{(1)}}
$$

to the first degree of approximation, as

$$
\operatorname{MSE}\left(\hat{Y}_{R}\right)=\left(\frac{g}{n}\right) Y^{2}\left[C_{y}^{2}+C_{x}^{2}(1-2 k)\right]
$$

From (3.23), (3.24), and (4.5) we have

$$
\begin{aligned}
& \operatorname{MSE}\left\{d_{r(1)}\right\}-\operatorname{MSE}\left(\hat{Y}_{R}\right)=\left(\frac{g}{n}\right) Y^{2} C_{x}^{2}(\lambda f-1)(\lambda f-2 k+1)<0, \quad \text { if } \\
& \min \left\{\frac{1}{f}, \frac{(2 k-1)}{f}\right\}<\lambda<\max \left\{\frac{1}{f}, \frac{(2 k-1)}{f}\right\} .
\end{aligned}
$$


(ii) $\operatorname{MSE}\left\{d_{r(2)}\right\}-\operatorname{MSE}\left(\hat{Y}_{R}\right)=\left(\frac{g}{n}\right) Y^{2} C_{x}^{2}(\lambda g-1)(\lambda g-2 k+1)<0, \quad$ if

$$
\min \left\{\frac{1}{g}, \frac{(2 \mathrm{k}-1)}{\mathrm{g}}\right\}<\lambda<\max \left\{\frac{1}{\mathrm{~g}}, \frac{(2 \mathrm{k}-1)}{\mathrm{g}}\right\} \text {. }
$$

Putting $(a, b)=(1,0) \Rightarrow \lambda=1$ in (3.23) and (3.24), we get the MSE of $\hat{Y}_{1}$ and $\hat{Y}_{2}$ to the first degree of approximation respectively as

$$
\begin{aligned}
& \operatorname{MSE}\left(\hat{Y}_{1}\right)=\left(\frac{g}{n}\right) Y^{2}\left[C_{y}^{2}+f C_{x}^{2}(f-2 k)\right], \\
& \operatorname{MSE}\left(\hat{Y}_{2}\right)=\left(\frac{g}{n}\right) Y^{2}\left[C_{y}^{2}+g C_{x}^{2}(g-2 k)\right] .
\end{aligned}
$$

From (3.23), (3.24), (4.6) and (4.7) we have

(iii) $\operatorname{MSE}\left\{\mathrm{d}_{\mathrm{r}(1)}\right\}-\operatorname{MSE}\left(\hat{\mathrm{Y}}_{1}\right)=\left(\frac{\mathrm{g}}{\mathrm{n}}\right) \mathrm{f} \mathrm{Y}^{2} \mathrm{C}_{\mathrm{x}}^{2}(1-\lambda)[\lambda f+f-2 \mathrm{k}]<0, \quad$ if

$$
\lambda>\frac{(2 k-f)}{f} \text {. }
$$

(iv) $\operatorname{MSE}\left\{d_{r(1)}\right\}-\operatorname{MSE}\left(\hat{Y}_{2}\right)=\left(\frac{g}{n}\right) Y^{2} C_{x}^{2}(\lambda f-g)[\lambda f+g-2 k]<0, \quad$ if

$$
\min \left\{\frac{f}{g}, \frac{(2 k-g)}{f}\right\}<\lambda<\max \left\{\frac{f}{g}, \frac{(2 k-g)}{f}\right\} \text {. }
$$

(iv) $\operatorname{MSE}\left\{d_{r(2)}\right\}-\operatorname{MSE}\left(\hat{Y}_{1}\right)=\left(\frac{g}{n}\right) Y^{2} C_{x}^{2}(\lambda g-f)[\lambda g+f-2 k]<0, \quad$ if

$$
\min \left\{\frac{f}{g}, \frac{(2 k-f)}{g}\right\}<\lambda<\max \left\{\frac{f}{g}, \frac{(2 k-f)}{g}\right\} \text {. }
$$

(v) $\operatorname{MSE}\left\{d_{r(2)}\right\}-\operatorname{MSE}\left(\hat{Y}_{2}\right)=-\left(\frac{g^{2}}{n}\right) Y^{2} C_{x}^{2}(1-\lambda)[\lambda g+g-2 k]<0, \quad$ if

$$
\lambda>\frac{(2 \mathrm{k}-\mathrm{g})}{\mathrm{g}} \text {. }
$$

Minimization of $\operatorname{MSE}\left(d_{r}\right), \operatorname{MSE}\left\{d_{r(1)}\right\}$ and $\operatorname{MSE}\left\{d_{r(2)}\right\}$ respectively at (3.22),

(3.23) and (3.24) yield the optimum values of $\lambda$ as

$$
\begin{aligned}
& \lambda_{\mathrm{opt}}^{(0)}=\mathrm{k}, \\
& \lambda_{\mathrm{opt}}^{(1)}=(\mathrm{k} / \mathrm{f}), \\
& \lambda_{\mathrm{opt}}^{(2)}=(\mathrm{k} / \mathrm{g}),
\end{aligned}
$$

and thus the common minimum MSE of $d_{r}, d_{r(1)}$ and $d_{r(2)}$ is given by

$$
\operatorname{MSE}_{\text {min }}\left(d_{r}\right)=\operatorname{MSE}_{\text {min }}\left\{d_{r(1)}\right\}=\operatorname{MSE}_{\text {min }}\left\{d_{r(2)}\right\}=\frac{g}{n} S_{y}^{2}\left(1-\rho^{2}\right) \text {. }
$$




\section{Numerical example}

To illustrate our results we consider two population data sets.

Population I: [Das (1988)].

$y=$ Number of agricultural labourers for 1961.

$\mathrm{x}=$ Number of agricultural labourers for 1971 .

Population II: [Cochran (1977, p.325)].

$y=$ Number of persons per block.

$x=$ Number of rooms per block.

The descriptions of population data sets I and II are given in Table 5.1.

\section{Table 5.1: Data Statistics}

\begin{tabular}{|c|c|c|}
\hline \multirow{2}{*}{ Parameter } & \multicolumn{2}{|c|}{ Population } \\
\hline & I & II \\
\hline $\mathrm{N}$ & 278 & 100 \\
\hline $\mathrm{n}$ & 30 & 10 \\
\hline Y & 10860.90 & 10110 \\
\hline$x$ & 6980.68 & 5880 \\
\hline$C_{y}$ & 1.445 & 0.145 \\
\hline$C_{x}$ & 1.6198 & 0.1281 \\
\hline $\bar{\rho}$ & 0.7213 & 0.6500 \\
\hline
\end{tabular}

We have computed Var/mean square errors (MSEs) of the estimators $\hat{Y}_{,} \hat{Y}_{R}, \hat{Y}_{1}$, $\hat{Y}_{2}, d_{i}$ and $d_{i(j)},(i=1,2, \ldots, 6 . ; j=1,2, \ldots, 12$.) using above two population data sets for different values of ' $a$ ' and ' $b$ '.

To asses, the performance of the estimators $\hat{Y}_{R}, \hat{Y}_{1}, \hat{Y}_{2}, d_{i}, d_{i(j)},(i=1,2, \ldots, 6$; $j=1,2, \ldots, 12$.) and regarding the biasedness of the estimators we have computed the following quantities

$$
B_{1}=\left|\frac{B\left(\hat{Y}_{1}\right)}{\left(\frac{g}{n}\right) Y C_{x}^{2}}\right|, I=0,1,2 ., B_{i}^{*}=\left|\frac{B\left(d_{i}\right)}{\left(\frac{g}{n}\right) Y C_{x}^{2}}\right|, B_{i(j)}=\left|\frac{B\left\{d_{i(j)}\right\}}{\left(\frac{g}{n}\right) Y C_{x}^{2}}\right| \begin{aligned}
& i=1,2, \ldots, 6 . \\
& j=1,2, \ldots, 12 .
\end{aligned}
$$

for different values of ' $a$ ' and ' $b$ ', here we designate $\hat{Y}_{0}=\hat{Y}_{R}$. Findings are displayed in Table 5.2. 
Table 5.2: Var/MSEs of different estimators

\begin{tabular}{|l|c|c|c|c|c|}
\hline \multirow{2}{*}{ Estimator } & \multicolumn{2}{|c|}{ Var/MSE } & \multirow{2}{*}{$\begin{array}{c}\text { Values of the } \\
\text { quantities }\end{array}$} & \multicolumn{2}{|c|}{ Population } \\
\cline { 2 - 3 } & Population & II & I & II \\
\hline$\hat{Y}$ & 7325091.64 & 193410.85 & - & - & - \\
\hline$\hat{Y}_{R}$ & 4683659.99 & 122235.29 & $B_{0}$ & 0.37 & 0.26 \\
\hline$\hat{Y}_{1}$ & 6154066.13 & 172707.48 & $B_{1}$ & 0.07 & 0.07 \\
\hline$\hat{Y}_{2}$ & 4082727.64 & 115767.03 & $B_{2}$ & 0.32 & 0.24 \\
\hline$d_{1}$ & 4319822.79 & 122062.58 & $B_{1}^{*}$ & 0.28 & 0.26 \\
\hline$d_{1(1)}$ & 6218927.10 & 172749.21 & $B_{1(1)}$ & 0.06 & 0.07 \\
\hline$d_{1(2)}$ & 3862277.42 & 115670.59 & $B_{1(2)}$ & 0.25 & 0.23 \\
\hline$d_{2}$ & 4207018.95 & 121970.48 & $B_{2}^{*}$ & 0.24 & 0.26 \\
\hline$d_{2(1)}$ & 6242120.24 & 172771.61 & $B_{2(1)}$ & 0.06 & 0.07 \\
\hline$d_{2(2)}$ & 3797012.88 & 115619.32 & $B_{2(2)}$ & 0.22 & 0.23 \\
\hline$d_{3}$ & 4365523.84 & 120299.31 & $B_{3}^{*}$ & 0.29 & 0.23 \\
\hline$d_{3(1)}$ & 6210023.22 & 173197.85 & $B_{3(1)}$ & 0.06 & 0.07 \\
\hline$d_{3(2)}$ & 3889211.06 & 114708.62 & $B_{3(2)}$ & 0.26 & 0.21 \\
\hline$d_{4}$ & 3583593.57 & 113828.75 & $B_{4}^{*}$ & 0.06 & 0.10 \\
\hline$d_{4(1)}$ & 6448625.01 & 175529.17 & $B_{4(1)}$ & 0.05 & 0.06 \\
\hline$d_{4(2)}$ & 3514644.50 & 111863.49 & $B_{4(2)}$ & 0.06 & 0.09 \\
\hline$d_{5}$ & 3623019.91 & 114305.22 & $B_{5}^{*}$ & 0.08 & 0.11 \\
\hline$d_{5(1)}$ & 6424130.00 & 175281.93 & $B_{5(1)}$ & 0.05 & 0.06 \\
\hline$d_{5(2)}$ & 3521066.55 & 111997.43 & $B_{5(2)}$ & 0.07 & 0.10 \\
\hline$d_{6}$ & 4420055.75 & 120807.65 & $B_{6}^{*}$ & 0.30 & 0.24 \\
\hline$d_{6(1)}$ & 6199724.34 & 173063.97 & $B_{6(1)}$ & 0.07 & 0.07 \\
\hline$d_{6(2)}$ & 3921674.61 & 114981.41 & $B_{6(2)}$ & 0.27 & 0.22 \\
\hline
\end{tabular}


It is observed from Table 5.2 that the proposed estimators $d_{4}$ and $d_{5}$ (which only utilize information on population total $X$ of the auxiliary variable $x$ like ratio estimator) are better than the usual unbiased estimator $\hat{Y}$, ratio estimator $\hat{Y}_{R}$, Srivenkataramana and Tracy's (1979) estimators $\hat{Y}_{1}$ and $\hat{Y}_{2}$, Sisodia and Dwivedi's (1981) estimator $d_{1}$, Kadilar and Cingi (2008) estimator $d_{2}$ and the proposed estimators $d_{1(1)}, d_{1(2)}, d_{2(1)}, d_{2(2)}, d_{3}, d_{3(1)}, d_{3(2)}, d_{4(1)}, d_{5(1)}, d_{6}, d_{6(1)}$ and $d_{6(2)}$ in the sense of having least MSEs among all of them for both the population data sets I and II. However, the modified proposed estimators $d_{4(2)}$ and $d_{5(2)}$ are more efficient than $d_{4}$ and $d_{5}$ with maximum reduction in MSEs for both the population data sets.

Further it is observed that the magnitude of the biases of the estimators $d_{4(2)}$ and $d_{5(2)}$ are also not appreciable. Thus the proposed modified estimators $d_{4(2)}$ and $d_{5(2)}$ are recommended for their use in practice.

\section{Estimator for the case of negative correlation}

When the correlation between the study variate $\mathrm{y}$ and the auxiliary variate $\mathrm{x}$ is negative, Robson (1957) suggested the use of product method of estimation which was revisited by Murthy (1964). The classical product estimator for the population total $\mathrm{Y}$ is

$$
\hat{Y}_{P}=\hat{Y} \frac{\hat{X}}{X}=Y^{(1)} \frac{X^{(1)}}{f^{2} X} \text {. }
$$

The exact expressions for the bias and mean squared error of the product estimator $\hat{Y}_{p}$ are as follows

$$
\begin{aligned}
& B\left(\hat{Y}_{P}\right)=Y V_{11}, \\
& \operatorname{MSE}\left(\hat{Y}_{P}\right)=Y^{2}\left[V_{20}+2 V_{11}+V_{02}+2 V_{21}+2 V_{12}+V_{22}\right],
\end{aligned}
$$

where, $V_{i j}(i, j=1,2,3,4)$ are same as defined earlier. 
In view of the identities (2.1) and (2.2), Srivenkataramana and Tracy (1979) proposed the following alternatives to the product estimator $\hat{Y}_{p}$.

$$
\begin{aligned}
\hat{Y}_{3} & =Y^{(1)}+\left\{\hat{Y}-Y^{(1)}\right\} g \frac{X}{X^{(2)}} \\
& =Y^{(1)}\left\{1+g^{2} \frac{X}{f X^{(2)}}\right\}, \\
\hat{Y}_{4} & =Y^{(1)}+\left\{\hat{Y}-Y^{(1)}\right\} g \frac{\hat{X}}{X} \\
& =Y^{(1)}\left\{1+\frac{g X^{(1)}}{f^{2} X}\right\} .
\end{aligned}
$$

The bias and MSE of these estimators are provided by

$$
\begin{aligned}
& B\left(\hat{Y}_{3}\right)=f Y\left[V_{11}+h V_{02}+h V_{12}+h^{2} V_{03}+h^{2} V_{13}+h^{3} V_{04}\right] \text {, } \\
& \mathrm{B}\left(\hat{\mathrm{Y}}_{4}\right)=\mathrm{gB}\left(\hat{\mathrm{Y}}_{\mathrm{p}}\right) \text {, } \\
& \operatorname{MSE}\left(\hat{Y}_{3}\right)=\mathrm{Y}^{2}\left[\mathrm{~V}_{02}+\mathrm{f}\left\{2 \mathrm{~V}_{11}+\mathrm{f} \mathrm{V}_{02}+2 \mathrm{~V}_{21}+2(\mathrm{f}+\mathrm{h}) \mathrm{V}_{12}+2 \mathrm{fhV} \mathrm{V}_{03}\right.\right. \\
& \left.\left.+(f+2 h) V_{22}+2 h(2 f+h) V_{13}+3 f h^{2} V_{04}\right\}\right] \\
& \operatorname{MSE}\left(\hat{\mathrm{Y}}_{4}\right)=\mathrm{Y}^{2}\left[\mathrm{~V}_{02}+\mathrm{g}\left\{2 \mathrm{~V}_{11}+\mathrm{gV_{02 }}+2 \mathrm{~V}_{21}+2 \mathrm{gV_{12 }}+\mathrm{gV_{22 }}\right\}\right] \text {, }
\end{aligned}
$$

where $h=f / g$.

Introducing the following transformation on the auxiliary variable $x$

$$
Z_{i}=a X_{i}+b, i=1,2, \ldots, N
$$

on the lines of Singh and Agnihotri (2008) we define the following product-type estimator for population total $\mathrm{Y}$ as

$$
t_{p}=\hat{Y} \frac{\hat{Z}}{Z}=Y^{(1)} \frac{Z^{(1)}}{f^{2} Z}=Y^{(1)} \frac{\left\{a X^{(1)}+n b\right)}{f^{2}(a X+N b)},
$$

where $(a, b)$ are same as defined in section 2. Some members of the estimator $t_{p}$ are shown in Table 6.1. 
Table 6.1: Some estimators of the population total $Y(=N \bar{Y})$ based on transformed auxiliary variable $x$

\begin{tabular}{|c|c|c|}
\hline \multirow{2}{*}{ Estimator } & \multicolumn{2}{|c|}{ Values of constants } \\
\cline { 2 - 3 } & $a$ & $b$ \\
\hline$t_{1}=Y^{(1)} \frac{\left\{X^{(1)}+n C_{x}\right\}}{f^{2}\left(X+N C_{x}\right)}$ & 1 & $C_{x}$ \\
\hline$t_{2}=Y^{(1)} \frac{\left\{X^{(1)} p+n C_{x}\right\}}{f^{2}\left(X p+N C_{x}\right)}$ & $\rho$ & $C_{x}$ \\
\hline$t_{3}=Y^{(1)} \frac{\left\{X^{(1)} p+n\right\}}{f^{2}(X p+N)}$ & $\rho$ & 1 \\
\hline$t_{4}=Y^{(1)} \frac{\left\{X^{(1)} f+n\right\}}{f^{2}(X f+N)}$ & $f$ & 1 \\
\hline$t_{5}=Y^{(1)} \frac{\left\{X^{(1)} f+n g\right\}}{f^{2}(X f+N g)}$ & $f$ & $g$ \\
\hline$t_{6}=Y^{(1)} \frac{\left\{X^{(1)} g+n\right\}}{f^{2}(X g+N)}$ & $g$ & 1 \\
\hline
\end{tabular}

Keeping the identities (2.1) and (2.2) in view, we define the following modified versions of $t_{p}$ respectively as

$$
\begin{aligned}
& t_{p(1)}=Y^{(1)}\left[1+\frac{g^{2}}{f} \frac{(a X+N b)}{\left\{a X^{(2)}+(N-n) b\right\}}\right], \\
& t_{p(2)}=Y^{(1)}\left[1+\frac{g}{f^{2}} \frac{\left\{a X^{(1)}+n b\right\}}{(a X+N b)}\right],
\end{aligned}
$$

where $f=n / N$ is the sampling fraction and $g=(1-f)$.

For $(a, b)=(1,0), t_{p(1)}$ and $t_{p(2)}$ respectively reduce to

$$
\begin{aligned}
& \hat{Y}_{3}=Y^{(1)}\left\{1+g^{2} \frac{X}{f X^{(2)}}\right\}, \\
& \hat{Y}_{4}=Y^{(1)}\left\{1+\frac{g X^{(1)}}{f^{2} X}\right\},
\end{aligned}
$$

which are due to Srivenkataramana and Tracy (1979). 
Table 6.2: Some members of the estimators $t_{p(1)}$ and $t_{p(2)}$

\begin{tabular}{|c|c|c|}
\hline \multirow{2}{*}{ Estimators } & \multicolumn{2}{|c|}{ Values of constants } \\
\hline & $a$ & $\mathrm{~b}$ \\
\hline$t_{1(1)}=Y^{(1)}\left[1+\frac{g^{2}}{f} \frac{\left(X+N C_{x}\right)}{\left\{X^{(2)}+(N-n) C_{x}\right\}}\right]$ & 1 & $C_{x}$ \\
\hline$t_{1(2)}=Y^{(1)}\left[1+\frac{g}{f^{2}} \frac{\left\{X^{(1)}+n C_{x}\right\}}{\left(X+N C_{x}\right)}\right]$ & 1 & $C_{x}$ \\
\hline$t_{2(1)}=Y^{(1)}\left[1+\frac{g^{2}}{f} \frac{\left(X \rho+N C_{x}\right)}{\left\{X^{(2)} \rho+(N-n) C_{x}\right\}}\right]$ & $\rho$ & $C_{x}$ \\
\hline$t_{2(2)}=Y^{(1)}\left[1+\frac{g}{f^{2}} \frac{\left\{X^{(1)} p+n C_{x}\right\}}{\left(X p+N C_{x}\right)}\right]$ & $\rho$ & $C_{x}$ \\
\hline$t_{3(1)}=Y^{(1)}\left[1+\frac{g^{2}}{f} \frac{(X \rho+N)}{\left\{X^{(2)} \rho+(N-n)\right\}}\right]$ & $\rho$ & 1 \\
\hline$t_{3(2)}=Y^{(1)}\left[1+\frac{g}{f^{2}} \frac{\left\{X^{(1)} \rho+n\right\}}{(X \rho+N)}\right]$ & $\rho$ & 1 \\
\hline$t_{4(1)}=Y^{(1)}\left[1+\frac{g^{2}}{f} \frac{(X f+N)}{\left\{X^{(2)} f+(N-n)\right\}}\right]$ & $f$ & 1 \\
\hline$t_{4(2)}=Y^{(1)}\left[1+\frac{g}{f^{2}} \frac{\left\{X^{(1)} f+n\right\}}{(X f+N)}\right]$ & $f$ & 1 \\
\hline$t_{5(1)}=Y^{(1)}\left[1+\frac{g^{2}}{f} \frac{(X f+N g)}{\left\{X^{(2)} f+(N-n) g\right\}}\right]$ & $\mathrm{f}$ & g \\
\hline$t_{5(2)}=Y^{(1)}\left[1+\frac{g}{f^{2}} \frac{\left\{X^{(1)} f+n g\right\}}{(X f+N g)}\right]$ & $f$ & g \\
\hline$t_{6(1)}=Y^{(1)}\left[1+\frac{g^{2}}{f} \frac{(X g+N)}{\left\{X^{(2)} g+(N-n)\right\}}\right]$ & g & 1 \\
\hline$t_{6(2)}=Y^{(1)}\left[1+\frac{g}{f^{2}} \frac{\left\{X^{(1)} g+n\right\}}{(X g+N)}\right]$ & g & 1 \\
\hline
\end{tabular}

In order to consider the efficiencies of these estimators in comparison to those of the unbiased estimator $\hat{Y}$, usual product estimator $\hat{Y}_{p}$, Srivenkataramana and Tracy (1979) estimators $\hat{Y}_{3}, \hat{Y}_{4}$, product-type estimator $t_{p}$ and $t_{p(j)}(j=1,2)$. The expressions for the bias and the mean square error (MSE) of the estimators are given in the following section. 


\section{Bias and MSE of the estimators $t_{p}$ and $t_{p(j)}(j=1,2)$}

Approximate expressions for the bias and mean squared error of the estimators $t_{p}, t_{p(1)}$ and $t_{p(2)}$ are respectively given by

$$
\begin{aligned}
& \mathrm{B}\left(\mathrm{t}_{\mathrm{p}}\right)=\mathrm{Y \lambda} \mathrm{V}_{11} \text {, } \\
& B\left\{t_{p(1)}\right\}=f Y\left[\lambda V_{11}+h \lambda^{2}\left(V_{02}+V_{12}\right)+h^{2} \lambda^{3}\left(V_{03}+V_{13}\right)+h^{3} \lambda^{4} V_{04}\right] \text {, } \\
& \mathrm{B}\left\{\mathrm{t}_{\mathrm{p}(2)}\right\}=\mathrm{Yg} \lambda \mathrm{V}_{11} \text {, } \\
& \operatorname{MSE}\left(\mathrm{t}_{\mathrm{p}}\right)=\mathrm{Y}^{2}\left[\mathrm{~V}_{20}+2 \lambda \mathrm{V}_{11}+\lambda^{2} \mathrm{~V}_{02}+2 \lambda \mathrm{V}_{21}+2 \lambda^{2} \mathrm{~V}_{12}+2 \lambda^{2} \mathrm{~V}_{22}\right] \\
& \operatorname{MSE}\left\{\mathrm{t}_{\mathrm{p}(1)}\right\}=\mathrm{Y}^{2}\left[\mathrm{~V}_{20}+\mathrm{f}\left\{2 \lambda \mathrm{V}_{11}+\lambda^{2} \mathrm{fV}_{02}+2 \lambda \mathrm{V}_{21}+2 \lambda^{2}(\mathrm{~h}+\mathrm{f}) \mathrm{V}_{12}+2 \lambda^{3} \mathrm{hfV} \mathrm{V}_{03}\right.\right. \\
& \left.\left.+(f+2 h) V_{22}+2 h \lambda^{3}(2 f+h) V_{13}+3 h^{2} f \lambda^{2} V_{04}\right\}\right] \\
& \operatorname{MSE}\left\{\mathrm{t}_{\mathrm{p}(2)}\right\}=\mathrm{Y}^{2}\left[\mathrm{~V}_{20}+\lambda g\left\{2 \mathrm{~V}_{11}+\lambda g \mathrm{~V}_{02}+2 \mathrm{~V}_{21}+2 \mathrm{~g} \lambda \mathrm{V}_{12}+g \lambda \mathrm{V}_{22}\right\}\right] \text {. }
\end{aligned}
$$

To have tangible idea about the biases and MSEs of the estimators $t_{p}, t_{p(1)}, t_{p(2)}$ and the estimators belonging to these estimators, we neglect the terms $V_{i j}$ with $\mathrm{i}+\mathrm{j}>2$ in $(7.1)-(7.6)$.

$$
\begin{aligned}
& \mathrm{B}\left(\mathrm{t}_{\mathrm{p}}\right) \approx \mathrm{Y} \lambda \mathrm{V}_{11}, \\
& \mathrm{~B}\left\{\mathrm{t}_{\mathrm{p}(1)}\right\} \approx \mathrm{f}\left[\lambda \mathrm{V}_{11}+\mathrm{h} \lambda^{2}\left(\mathrm{~V}_{02}+\mathrm{V}_{12}\right)\right], \\
& \mathrm{B}\left\{\mathrm{t}_{\mathrm{p}(2)}\right\} \approx \mathrm{Yg} \lambda \mathrm{V}_{11}=\mathrm{gB}\left(\mathrm{t}_{\mathrm{p}}\right), \\
& \operatorname{MSE}\left(\mathrm{t}_{\mathrm{p}}\right) \approx \mathrm{Y}^{2}\left[\mathrm{~V}_{20}+2 \lambda \mathrm{V}_{11}+\lambda^{2} \mathrm{~V}_{02}\right], \\
& \operatorname{MSE}\left\{\mathrm{t}_{\mathrm{p}(1)}\right\} \approx \mathrm{Y}^{2}\left[\mathrm{~V}_{20}+\mathrm{f}\left\{2 \lambda \mathrm{V}_{11}+\lambda^{2} f \mathrm{~V}_{02}\right\}\right], \\
& \operatorname{MSE}\left\{\mathrm{t}_{\mathrm{p}(2)}\right\} \approx \mathrm{Y}^{2}\left[\mathrm{~V}_{20}+\lambda g\left\{2 \mathrm{~V}_{11}+\lambda g \mathrm{~V}_{02}\right\}\right] .
\end{aligned}
$$

In case of simple random sampling without replacement (SRSWOR) $V_{11}=\left(\frac{g}{n}\right) k C_{x}^{2}, V_{20}=\left(\frac{g}{n}\right) C_{y}^{2}$ and $V_{02}=\left(\frac{g}{n}\right) C_{x}^{2}$ with $k=\rho\left(\frac{C_{y}}{C_{x}}\right)$ and the expressions (7.7)-(7.12) respectively reduce to

$$
\begin{aligned}
& B\left(t_{p}\right)=\left(\frac{g}{n}\right) Y \lambda k C_{x}^{2}, \\
& B\left\{t_{p(1)}\right\}=\left(\frac{g}{n}\right) Y \lambda f C_{x}^{2}(k+h \lambda), \\
& B\left\{t_{p(2)}\right\}=\left(\frac{g^{2}}{n}\right) Y \lambda k C_{x}^{2},
\end{aligned}
$$




$$
\begin{aligned}
& \operatorname{MSE}\left(t_{p}\right)=\left(\frac{g}{n}\right) Y^{2}\left[C_{y}^{2}+\lambda C_{x}^{2}\{\lambda+2 k\}\right], \\
& \operatorname{MSE}\left\{t_{p(1)}\right\}=\left(\frac{g}{n}\right) Y^{2}\left[C_{y}^{2}+\lambda f C_{x}^{2}\{\lambda f+2 k\}\right], \\
& \operatorname{MSE}\left\{t_{p(2)}\right\}=\left(\frac{g}{n}\right) Y^{2}\left[C_{y}^{2}+\lambda g C_{x}^{2}\{\lambda g+2 k\}\right] .
\end{aligned}
$$

\section{Efficiency comparison}

The approximate reductions in the MSE of the estimator $t_{p(1)}$ or $t_{p(2)}$ is used in the place of $t_{p}$, up to second order moments, given by

$$
\begin{aligned}
& \operatorname{MSE}\left(t_{p}\right) \quad \operatorname{MSE}\left\{t_{p(1)}=Y^{2} \frac{(1-f)}{n} g \lambda V_{02}\left[(1+f) \lambda-2 k^{\prime}\right],\right. \\
& \operatorname{MSE}\left(t_{p}\right) \quad \operatorname{MSE}\left\{t_{p(2)}\right\}=Y^{2} \frac{(1-f)}{n}(1-g) \lambda V_{02}\left[(1+g) \lambda-2 k^{\prime}\right],
\end{aligned}
$$

where $k^{\prime}=-k$. Note that $k^{\prime}$ is positive since $k$ is now negative. The guidelines for choosing among $\hat{Y}, t_{p}, t_{p(1)}, t_{p(2)}$ are in Table 8.1.

Table 8.1: The case of negative correlation

\begin{tabular}{|l|c|c|c|}
\hline \multicolumn{2}{|c|}{$f \leq \frac{1}{2}$} & \multicolumn{2}{c|}{$f>\frac{1}{2}$ or $g=(1-f)<\frac{1}{2}$} \\
\hline Interval for $\frac{k^{\prime}}{\lambda}=-\frac{V_{11}}{V_{02}}$ & $\begin{array}{c}\text { preferred } \\
\text { estimator }\end{array}$ & Interval for $\frac{k^{\prime}}{\lambda}=-\frac{V_{11}}{V_{02}}$ & $\begin{array}{c}\text { preferred } \\
\text { estimator }\end{array}$ \\
\hline$(1)$ & $(2)$ & $(3)$ & $(4)$ \\
\hline $0 \leq \frac{k^{\prime}}{\lambda} \leq \frac{f}{2}$ & $\hat{Y}$ & $0<\frac{k^{\prime}}{\lambda} \leq \frac{g}{2}$ & $\hat{Y}$ \\
\hline$\frac{f}{2}<\frac{k^{\prime}}{\lambda} \leq \frac{1}{2}$ & $t_{p(1)}$ & $\frac{g}{2}<\frac{k^{\prime}}{\lambda} \leq \frac{1}{2}$ & $t_{p(2)}$ \\
\hline$\frac{1}{2}<\frac{k^{\prime}}{\lambda} \leq\left(1-\frac{1}{2}\right)$ & $t_{p(2)}$ & $\frac{1}{2}<\frac{k^{\prime}}{\lambda} \leq\left(1-\frac{g}{2}\right)$ & $t_{p(1)}$ \\
\hline$k^{\prime}>\left(1-\frac{1}{2}\right)$ & $t_{p}$ & $\frac{k^{\prime}}{\lambda}>\left(1-\frac{g}{2}\right)$ & $t_{p}$ \\
\hline
\end{tabular}

It is observed from Table 4.1 and Table 8.1 that when the sampling fraction $f \leq \frac{1}{2}$ and the value of $\frac{k}{\lambda}\left(\frac{k^{\prime}}{\lambda}\right)$ is (a) very low it is better to use the simple estimator $\hat{Y}$, (b) temperately low it is efficient to use $d_{r(1)}\left(t_{p(1)}\right)$, (c) temperately large it is 
preferable to use $d_{r(2)}\left(t_{p(2)}\right)$ and (d) very large it is excellent to use $d_{r}\left(t_{p}\right)$ as an estimator of the population total $Y$. In the impending case of $f>\frac{1}{2}$ the acts of $d_{r(1)}\left(t_{p(1)}\right)$ and $d_{r(2)}\left(t_{p(2)}\right)$ are exchangeable with each other.

Now minimizing the mean square error of the estimators $t_{p}, t_{p(1)}$ and $t_{p(2)}$ with respect to $\lambda$ and equating them to zero we get the optimum value of $\lambda$ respectively as

$$
\begin{aligned}
& \lambda_{\mathrm{opt}}^{*(0)}=-\mathrm{k}, \\
& \lambda_{\mathrm{opt}}^{*(1)}=-(\mathrm{k} / \mathrm{f}), \\
& \lambda_{\mathrm{opt}}^{*(2)}=-(\mathrm{k} / \mathrm{g}),
\end{aligned}
$$

and thus the resulting common minimum MSE of $t_{p}, t_{p(1)}$ and $t_{p(2)}$ is given by

$$
\operatorname{MSE}_{\text {min }}\left(t_{p}\right)=\operatorname{MSE}_{\text {min }}\left\{t_{p(1)}\right\}=\operatorname{MSE}_{\text {min }}\left\{t_{p(2)}\right\}=\frac{g}{n} S_{y}^{2}\left(1-\rho^{2}\right) \text {. }
$$

\section{Acknowledgement}

Authors are thankful to the referees for their valuable suggestions regarding improvement of the earlier draft of the paper.

\section{References}

1. Bandyopadhyay, S. (1980). Improved ratio and product estimators. Sankhya, C, 42, 45-49.

2. Butt, N.S., Kamal, S. and Shahbaz, M.Q. (2010). Estimation of population mean in two phase sampling. Middle-East Jour. Sci. Res., 6(2), 198-200.

3. Cochran, W.G. (1977). Sampling Techniques. John Wiley and Sons, New-York, USA.

4. Das, A.K. (1988): Contributions to the theory of sampling strategies based on auxiliary information. Ph.D. thesis submitted to BCKV, Mohanpur, Nadia, West Bengal, India.

5. Gupta, S. and Shabbir, J. (2008). On improvement in estimating the population mean in simple random sampling. Jour. Appl. Stat., 35(5), 559-566.

6. Hanif, M., Shahbaz, M.Q. and Ahmad, Z. (2010). Some improved estimators in multiphase sampling. Pak. Jour. Stat., 26(1), 195-202.

7. Kadilar, C. and Cingi, H. (2006). An improvement in estimating the population mean by using the correlation coefficient. Hacett. Jour. Math. Stat., 35(1), 103-109. 
8. Khoshnevisan, M. Singh, R., Chauhan, P., Sawan, N. and Smarandache, F. (2007). A general family of estimators for estimating population mean using known value of some population parameter(s). Far. East. Jour. Theo. Stat., 22, 181-191.

9. Mohanty, S. and Sahoo, J. (1995). A note on improving the ratio method of estimators through linear transformation using certain known population parameters. Sankhya, B, 57(1), 93-102.

10. Murthy, M.N. (1967). Sampling theory and methods. Statistical Publishing Society, Calcutta, India.

11. Murthy, M.N. (1964). Product method of estimation. Sankhya, 26(A), 284-307.

12. Reddy, V.N. (1974). On a transformed ratio method of estimation. Sankhya, C, 36(1), 59-70.

13. Robson, D.S. (1957). Application of multivariate polykays to the theory of unbiased ratio-type estimators. Jour. Amer. Statist. Assoc., 52, 511-522.

14. Singh, G.N. (2003). On the improvement of product method of estimation in sample surveys. Jour. Ind. Soc. Agri. Stat., 56(3), 267-175.

15. Singh, H.P. and Agnihotri, N. (2008). A general procedure of estimating population mean using auxiliary information in sample surveys. Stat. Trans., 9(1), 71-87.

16. Singh, H.P. and Tailor, R. (2003). Use of known correlation coefficient in estimating the finite population mean. Stat. Trans., 6(4), 555-560.

17. Singh, H.P., Tailor, R., Tailor, R. and Kakran, M.S. (2004). An improved estimator of population mean using power transformation. Jour. Ind. Soc. Agri. Stat., 58(2), 223-230.

18. Singh, R., Kumar, M. and Smarandache, F. (2008). Almost unbiased estimator for estimating population mean using known value of some population parameter(s). Pak. Jour. Stat. Oper. Res., 4(2), 63-76.

19. Sisodia, B.V.S. and Dwivedi, V.K. (1981). A modified ratio estimator using coefficient of variation of auxiliary variable. Jour. Ind. Soc. Agri. Stat., 33(2), 13-18.

20. Srivenkataramana, T. and Tracy, D.S. (1979). On ratio and product methods of estimation in sampling. Statistica Neerlandica, 33(1), 37-49.

21. Srivenkataramana, T. and Tracy, D.S. (1980). An alternative to ratio method in sample surveys. Ann. Inst. Statist. Math., A, 32, 111-120.

22. Upadhyaya, L.N. and Singh, H.P. (1999). Use of transformed auxiliary variable in estimating the finite population mean. Biom. Jour., 41(5), 627-636. 